\title{
Injury enhances TLR2 function and antimicrobial peptide expression through a vitamin D-dependent mechanism
}

\author{
Jürgen Schauber, ${ }^{1}$ Robert A. Dorschner, ${ }^{1}$ Alvin B. Coda, ${ }^{1}$ Amanda S. Büchau, ${ }^{1}$ Philip T. Liu, ${ }^{2}$ \\ David Kiken, ${ }^{1}$ Yolanda R. Helfrich, ${ }^{3}$ Sewon Kang, ${ }^{3}$ Hashem Z. Elalieh, ${ }^{4}$ Andreas Steinmeyer, ${ }^{5}$ \\ Ulrich Zügel, ${ }^{6}$ Daniel D. Bikle, ${ }^{4}$ Robert L. Modlin, ${ }^{2}$ and Richard L. Gallo' \\ 1Division of Dermatology, University of California, San Diego, and VA San Diego Healthcare System, San Diego, California, USA \\ 2Division of Dermatology, Department of Medicine, David Geffen School of Medicine, University of California, Los Angeles, \\ Los Angeles, California, USA. ${ }^{3}$ Department of Dermatology, University of Michigan, Ann Arbor, Michigan, USA. \\ ${ }^{4}$ Department of Medicine, Endocrine Unit, Veterans Affairs Medical Center, University of California, San Francisco, San Francisco, California, USA. \\ ${ }^{5}$ Medicinal Chemistry and ${ }^{6}$ Corporate Research Business Area Inflammation, Schering AG, Berlin, Germany.
}

\begin{abstract}
An essential element of the innate immune response to injury is the capacity to recognize microbial invasion and stimulate production of antimicrobial peptides. We investigated how this process is controlled in the epidermis. Keratinocytes surrounding a wound increased expression of the genes coding for the microbial pattern recognition receptors CD14 and TLR2, complementing an increase in cathelicidin antimicrobial peptide expression. These genes were induced by $1,25(\mathrm{OH})_{2}$ vitamin $\mathrm{D}_{3}(1,25 \mathrm{D} 3$; its active form), suggesting a role for vitamin $D_{3}$ in this process. How $1,25 \mathrm{D} 3$ could participate in the injury response was explained by findings that the levels of CYP27B1, which converts $25 \mathrm{OH}$ vitamin $\mathrm{D}_{3}(25 \mathrm{D} 3)$ to active $1,25 \mathrm{D} 3$, were increased in wounds and induced in keratinocytes in response to TGF- $\beta_{1}$. Blocking the vitamin $D$ receptor, inhibiting CYP27B1, or limiting $25 \mathrm{D} 3$ availability prevented TGF- $\beta_{1}$ from inducing cathelicidin, CD14, or TLR2 in human keratinocytes, while CYP27B1-deficient mice failed to increase CD14 expression following wounding. The functional consequence of these observations was confirmed by demonstrating that 1,25D3 enabled keratinocytes to recognize microbial components through TLR2 and respond by cathelicidin production. Thus, we demonstrate what we believe to be a previously unexpected role for vitamin $D_{3}$ in innate immunity, enabling keratinocytes to recognize and respond to microbes and to protect wounds against infection.
\end{abstract}

\section{Introduction}

Innate immunity encompasses all mechanisms that resist infection without the need for prior exposure to the pathogen. The innate immune response is an evolutionarily ancient system that is an important part of mammalian immune defense. In the course of the analysis of this system, several unexpected associations have emerged to explain how factors not previously thought to contribute to the immune response may influence human disease. For example, recent insights into the functions of $1,25(\mathrm{OH})_{2}$ vitamin $\mathrm{D}_{3}(1,25 \mathrm{D} 3)$ as an immune-modifying agent have illuminated a large body of previously unexplained associations between alterations in vitamin $\mathrm{D}_{3}$ and infectious disease $(1,2)$. Elevated $1,25 \mathrm{D} 3$ and hypercalcemia have been associated with active pulmonary tuberculosis (3), and lower serum concentrations of the $1,25 \mathrm{D} 3$ precursor $25 \mathrm{OH}$ vitamin $\mathrm{D}_{3}$ (25D3) in African Americans correlates with increased susceptibility to infection (4). An explanation for these events has been provided by observations that stimulation of TLR2 increases production of $1,25 \mathrm{D} 3$ in monocytes, which in turn leads to an

Nonstandard abbreviations used: AMP, antimicrobial peptide; $\mathrm{Ct}$, threshold cycle; CYP24A1, 24-hydroxylase; CYP27B1, 1,25D3, 1,25(OH) 2 vitamin $\mathrm{D}_{3} ; 25 \mathrm{D} 3,25 \mathrm{OH}$ vitamin $\mathrm{D}_{3} ; 1 \alpha$-hydroxylase; qPCR, quantitative real-time RT-PCR; VDR, vitamin D receptor; VDRE, vitamin D-responsive element.

Conflict of interest: The authors have declared that no conflict of interest exists. Citation for this article: J. Clin. Invest. 117:803-811 (2007). doi:10.1172/JCI30142. increase in the production of antimicrobial peptides (AMPs) (4). Results of recent studies support a role for vitamin $\mathrm{D}_{3}$ in the regulation of innate immune functions (5).

Previous findings that $1,25 \mathrm{D} 3$ regulates the expression and activation of AMPs in monocytes and keratinocytes in the epidermis (6-8) suggest that in addition to its effects on differentiation and formation of a physical barrier $(9,10), 1,25 \mathrm{D} 3$ also provides a stimulus for rapid production of a chemical antimicrobial shield. In particular, 1,25D3 induces the expression of LL-37, a human AMP belonging to the cathelicidin family $(11,12)$. Cathelicidins are relevant to defense against microbes, as infections develop more easily in mice deficient in the cathelicidin gene $(13,14)$ and in humans with a deficiency in cathelicidin expression (15). With the observation that cathelicidin is increased with increasing concentrations of $1,25 \mathrm{D} 3(6-8)$, the importance of vitamin $\mathrm{D}_{3}$ to immune defense warrants renewed interest.

Vitamin $\mathrm{D}_{3}$ is produced from dietary or endogenous precursors under the influence of UVB light (16). Activation of vitamin $\mathrm{D}_{3}$ to $1,25 \mathrm{D} 3$ requires 2 major hydroxylation steps, the first by 25 -hydroxylase (CYP27A1) and the second by $1 \alpha$-hydroxylase (CYP27B1), enzymes located mainly in the human liver and kidney, respectively. However, some 1,25D3-targeted organs such as the epidermis also posses the enzymes to produce 1,25D3 (17). Upon binding to the vitamin D receptor (VDR), 1,25D3 activates target genes through vitamin D-responsive elements (VDREs) in the gene promoter (16). Simultaneously, 1,25D3 induces the vitamin $\mathrm{D}_{3}$ catabolic enzyme 

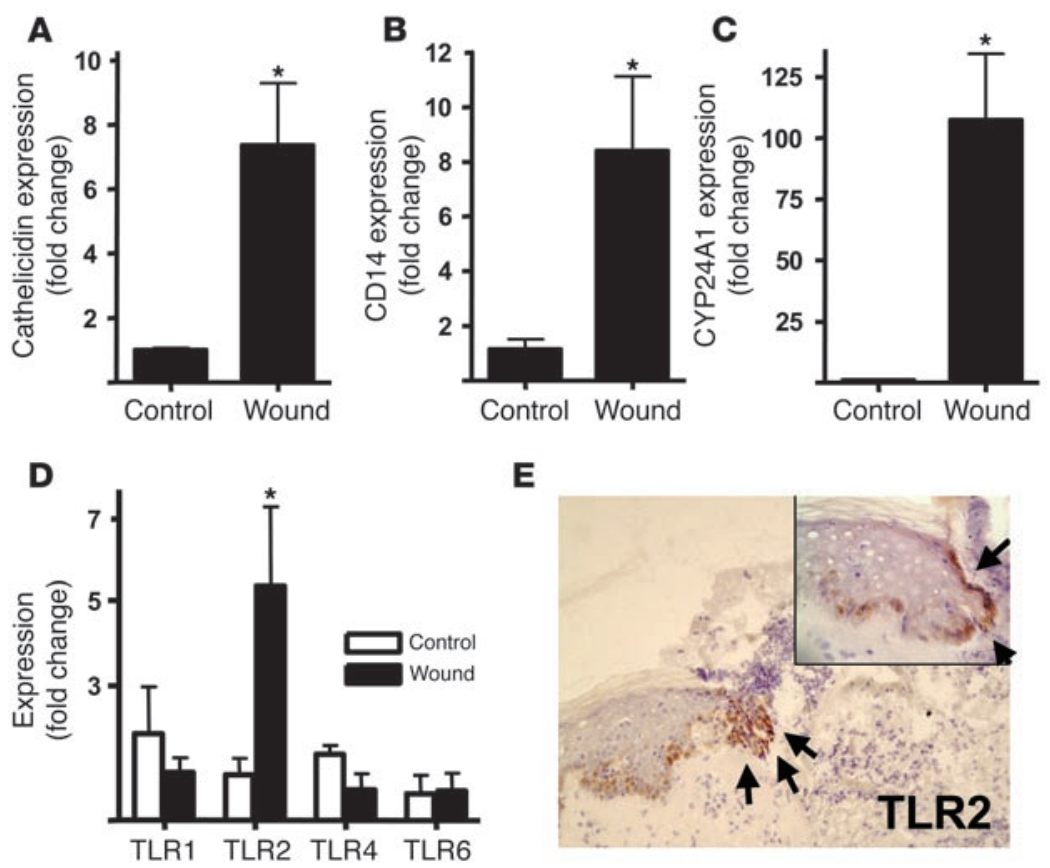

E

\section{Figure 1}

Injury triggers increased TLR2, CD14, and CYP24A1 in skin. Human wounds 24 hours after full-thickness sterile skin incision were evaluated for the expression of innate immune recognition and response molecules $(n=5)$. Transcript abundance was measured by qPCR, normalized to GAPDH expression, and compared with noninjured skin $(n=4)$. (A) Wounded skin showed an expected increase in expression of cathelicidin, a vitamin $D_{3}-$ responsive antimicrobial gene. Additional vitamin $D_{3}$-responsive genes, the TLR co-receptor CD14 (B) and the vitamin $D_{3}$ catabolic enzyme CYP24A1 (C), also increased after injury. (D) Expression of TLR2 mRNA, but not that of TLR1, TLR4, and TLR6, was increased after injury. (E) A corresponding increase in TLR2 protein staining on keratinocytes at the wound edges was seen (original magnification, $\times 100$ ). The incision site is marked by black arrows. An enlarged sector is displayed in the inset (original magnification, $\times 400) .{ }^{*} P<0.05$, Mann-Whitney test. 24-hydroxylase (CYP24A1), thereby initializing its own degradation. Control of 1,25D3-producing and -catabolizing enzymes therefore determines the level of bioactive hormone.

Control of cathelicidin expression follows a pattern consistent with expectations for a gene required for innate immune response, but these events are not known to involve alterations in $1,25 \mathrm{D} 3 \mathrm{lev}-$ els. Levels of cathelicidin, and several other AMPs, greatly increase after wounding or infection (18), but most classical signaling molecules active in the wound repair response - or microbial ligands that trigger pattern recognition events - have little or no effect on cathelicidin expression (11). Based on observations that cathelicidin is induced by $1,25 \mathrm{D} 3$ in vitro (6), we hypothesized that vitamin $\mathrm{D}_{3}$ signaling may be activated during skin injury. In this study, we investigated the expression of genes influenced by 1,25D3 and attempted to understand this in the setting of wound repair. We show for the first time to our knowledge that injury resulted in enhanced vitamin $\mathrm{D}_{3}$ metabolism and the subsequent induction of 1,25D3-regulated genes, which led to enhanced expression of essential elements of innate immunity. Our data support what we believe to be a previously unknown role for vitamin $\mathrm{D}_{3}$ in wound repair and provide insight into factors important to the control of the innate immune response in the skin.

\section{Results}

Injury triggers a local increase in 1,25D3 signaling in skin. The function of elements of cutaneous innate immunity, such as the expression of TLRs and the production of AMPs, is essential for control of infection $(13,19)$. The expression of AMPs such as the cathelicidin gene CAMP (and the peptide LL-37, which it encodes) increases dramatically in epithelia after injury or infection $(18,20)$. Recently, production of LL-37 was also shown to be induced by $1,25 \mathrm{D} 3$ (6-8) but not by several cytokines, growth factors, or other factors present in wounds (11). The findings that $1,25 \mathrm{D} 3$ stimulates cathelicidin expression and that cathelicidin increases following injury (18) prompted us to investigate whether the expression of other elements of innate immunity or 1,25D3-regulated genes are induced after wounding. RNA extracted from skin wound biopsies obtained after sterile full-thickness incision showed the expected increase in cathelicidin expression (Figure 1A). Coincident with this increase in cathelicidin, mRNA for the TLR cofactor CD14 and the 1,25D3 catabolic enzyme CYP24A1 were also induced (Figure 1, B and C). Both of these genes were previously shown to be inducible by $1,25 \mathrm{D} 3(4,21,22)$, but not known to change during the response to injury. This increase in mRNA was accompanied by an increase in CD14 protein expression in keratinocytes at the wound edge and in cells infiltrating the wound (Supplemental Figure 1; supplemental material available online with this article; doi:10.1172/JCI30142DS1). In addition, the expression of TLR2, not previously known to be influenced by $1,25 \mathrm{D} 3$, also increased as measured by transcript and protein abundance in keratinocytes (Figure 1, D and E). The expression of TLR1, TLR4, and TLR6 was not changed.

1,25D3 induces an increase in the expression of TLR2 and CD14. The finding that multiple, diverse genes under the control of $1,25 \mathrm{D} 3$ are increased after injury suggested that the abundance or bioavailability of $1,25 \mathrm{D} 3$ may increase in the wound and that these responses may be a previously unrecognized part of the human injury response. We also hypothesized that TLR2 expression is controlled by $1,25 \mathrm{D} 3$. To test this, the response of cultured human keratinocytes to $1,25 \mathrm{D} 3$ was studied. Similar to the response seen in vivo following injury, levels of CD14 and TLR2 mRNA and protein increased in cultured keratinocytes in response to 1,25D3 (Figure 2, A and B). Expression of other TLRs and the TLR coreceptor CD36 remained unchanged. TLR2 and CD14 transcript abundance was also induced by 1,25D3 in keratinocytes differentiated in epidermal constructs (Figure 2C). Patients' skin treated with topical 1,25D3 also showed increased protein expression of TLR2 (Figure 2D) and cathelicidin (Supplemental Figure 2). This increase in TLR2 seen in patients following a single application of 1,25D 3 was not associated with clinical or histological evidence of inflammation. Therefore, these observations show that 1,25D3 induces TLR2 in kerati- 
A

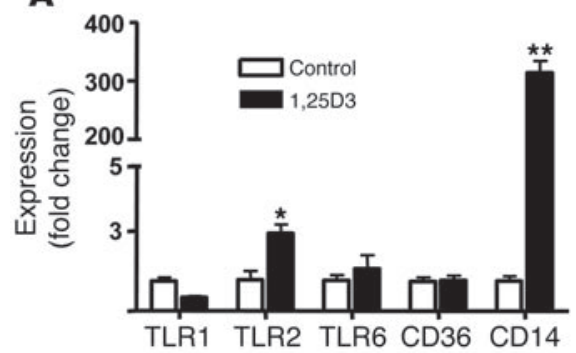

C

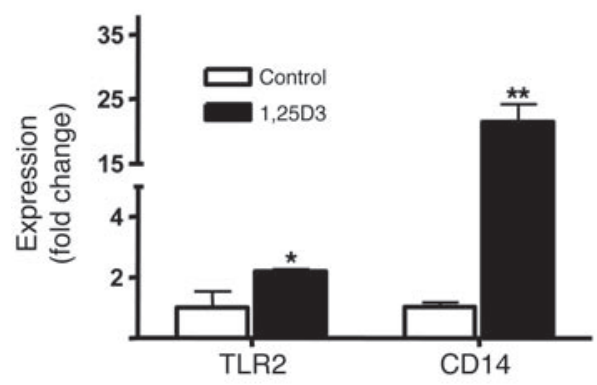

B
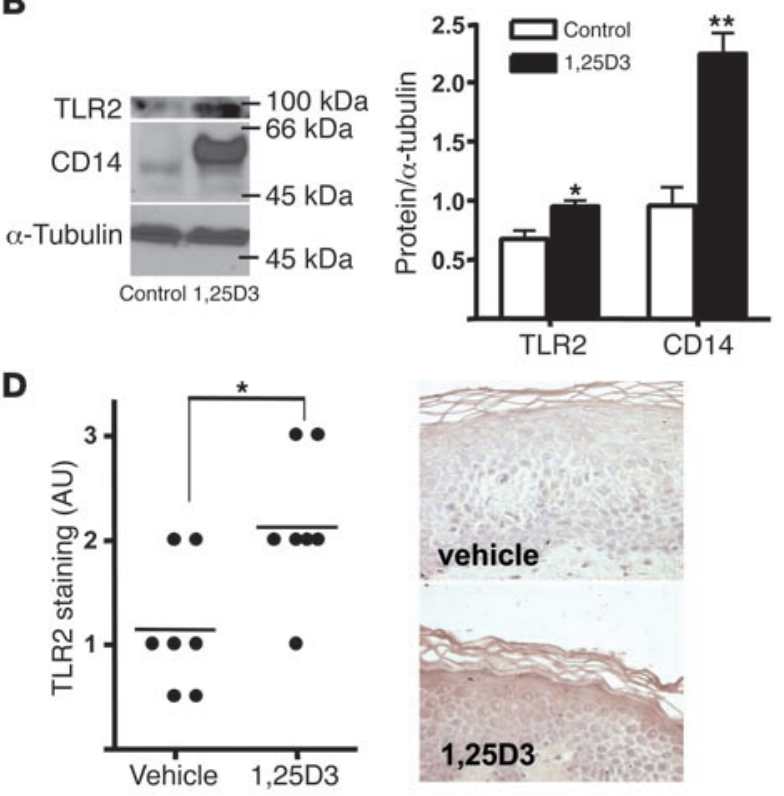

Figure 2

The effect of 1,25D3 on the expression of TLRs in cultured keratinocytes. (A) Expression of TLR2 and CD14 mRNA was increased by 1,25D3 $(100 \mathrm{nM})$ in cultured keratinocyte monolayers after 24 hours. (B) Expression of TLR2 and CD14 protein was increased by 1,25D3 (100 nM) in monolayer keratinocyte extracts evaluated by Western blot and quantified by image density analysis. (C) Keratinocytes grown in differentiated epidermal constructs stimulated with 1,25D3 (100 nM) also showed an increase in CD14 and TLR2 transcript abundance. Data are mean \pm SD of a representative experiment performed in triplicate. ${ }^{*} P<0.05,{ }^{* *} P<0.01$, Student's $t$ test. (D) Skin from healthy volunteers $(n=7)$ was treated with 1,25D3 (1.0 mM applied once). Controls are contralateral skin treated with vehicle. After 4 days, punch biopsies from both sites were obtained, and skin sections were stained for TLR2 expression. Staining intensity — graded according to the intensity of immunoreactivity ( 0 , no expression; 3, strong expression) - increased in patients treated with topical 1,25D3, as determined by an investigator blinded to treatment group. Sections from 1 representative study participant are displayed. ${ }^{*} P<0.05$, Mann-Whitney test.

nocytes in vitro and in vivo and that the same set of recognition and response elements of innate immunity are induced both by $1,25 \mathrm{D} 3$ and by injury.

CYP27B1 is induced by injury and by soluble factors found in wounds. The finding that genes expressed in injury were the same as those induced by $1,25 \mathrm{D} 3$ suggested that a mechanism exists in wounds that leads to an increase in available $1,25 \mathrm{D} 3$. To test this hypothesis, we next examined whether wounding, or soluble factors present in the wound, influence CYP27B1, the enzyme responsible for converting inactive $25 \mathrm{D} 3$ to active 1,25D3. The expression of this important enzyme following skin injury was to our knowledge previously unknown, but prior observations supported this hypothesis: in skin, the majority of vitamin $\mathrm{D}_{3}$ is stored in an inactive proform, 7-dehydrocholesterol (23), and some inflammatory mediators such as TNF- $\alpha$ and IFN- $\gamma$ influence $1,25 \mathrm{D} 3$ metabolism in monocytes and macrophages (24-26) as well as in nondifferentiated keratinocytes $(27,28)$.

Figure 3
In human skin, CYP27B1 expression increased after wounding (Figure 3A). Cultured keratinocytes exposed to TGF- $\beta_{1}$ also showed an increase in CYP27B1 expression, while TNF- $\alpha$ had no effect (Figure 3B). Also, similar to observations made in monocytes (4), CYP27B1 expression in keratinocytes could be induced by activation of TLR2 (Figure 3C). These data demonstrated that

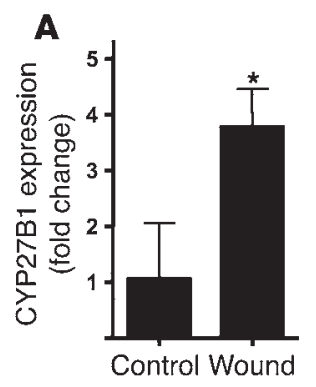

B
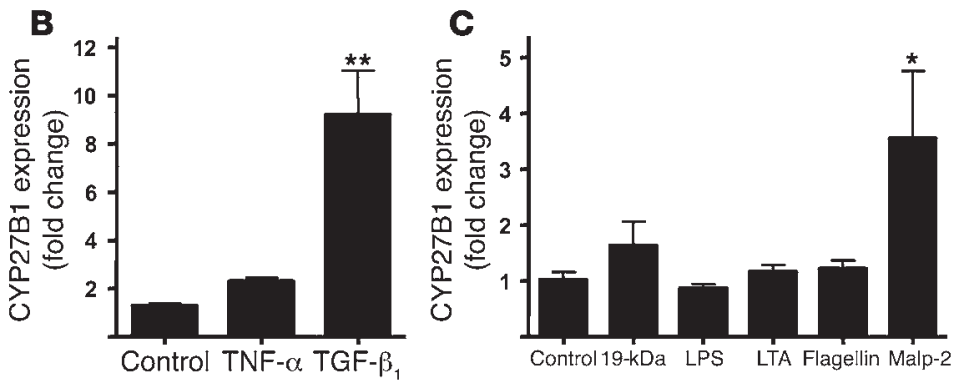

CYP27B1 is increased in response to injury, TGF- $\beta_{1}$, or activation of TLR2. (A) The expression of CYP27B1, which converts inactive 25D3 to active 1,25D3, was evaluated in skin wounds as in Figure 1. Wounded skin $(n=5)$ increased CYP27B1 mRNA compared with controls $(n=4) .{ }^{*} P<0.05$, Mann-Whitney test. (B) Keratinocytes were cultured in the presence of TNF- $\alpha(20 \mathrm{ng} / \mathrm{ml})$ or TGF- $\beta_{1}(1 \mu \mathrm{g} / \mathrm{ml})$ for 24 hours, after which RNA was isolated and CYP27B1 transcript abundance was analyzed by qPCR. (C) Keratinocytes were cultured with different TLR ligands for 24 hours, after which CYP27B1 expression analyzed as described above. Both TGF- $\beta_{1}$ and the TLR2 ligand Malp-2 induced CYP27B1 expression. 19-kDa, 19-kDa lipopeptide; LTA, lipoteichoic acid. Data are mean \pm SD of a single experiment performed in triplicate and are representative of 3 independent experiments. ${ }^{*} P<0.05$, ${ }^{* \star} P<0.01$, Student's $t$ test. 

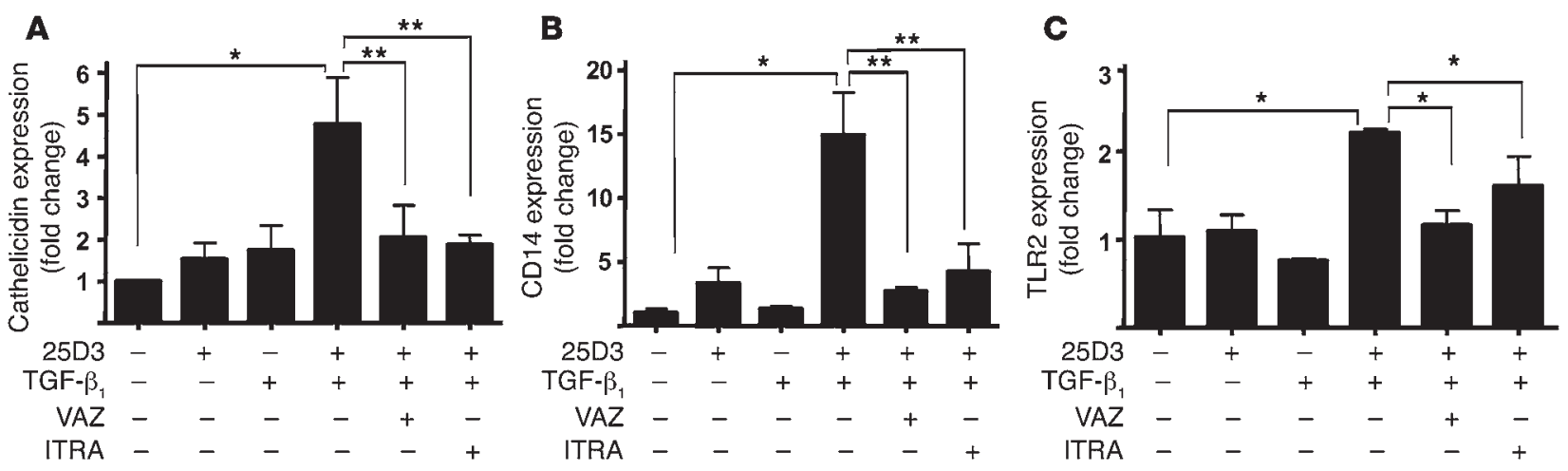

Figure 4

TGF- $\beta_{1}$ leads to an increase of 1,25D3-responsive genes in wounds by activation of CYP27B1. Keratinocytes were pretreated with the VDR antagonist ZK159222 (VAZ; $10^{-7} \mathrm{M}$ ) or the CYP27B1 antagonist itraconazole (ITRA; $10^{-7} \mathrm{M}$ ) and then stimulated with TGF- $\beta_{1}(1 \mathrm{ng} / \mathrm{ml})$ in the presence or absence of 25D3 (10 nM) for 24 hours. Expression of cathelicidin (A), CD14 (B), and TLR2 (C) mRNA was determined as described in Figure 2. Induction of these innate immune effector and response genes by TGF- $\beta_{1}$ found in wounds was dependent on the availability of $25 \mathrm{D} 3$ and the activity of CYP27B1 or a functional VDR. Data are mean \pm SD of a single experiment performed in triplicate and are representative of 3 independent experiments. ${ }^{*} P<0.05,{ }^{* \star} P<0.01$, Student's $t$ test.

injury leads to an increase in the expression of an enzyme capable of inducing a local increase in $1,25 \mathrm{D} 3$. This induction may occur secondary to the activity of factors present in the wound, such as IFN- $\gamma$ or TGF- $\beta_{1}$, or by microbial stimulation of TLR 2 .

TGF- $\beta_{1}$ induces cathelicidin, CD14, and TLR2 by CYP27B1-dependent activation of $1,25 D 3$. To directly test whether the stimulation of CYP27B1 is responsible for the induction of the innate immune molecules we observed in wounds, keratinocytes were next exposed to TGF- $\beta_{1}$ in the presence of $25 \mathrm{D} 3$. Prior studies with TGF- $\beta_{1}$, as well as several other molecules associated with wound repair, failed to show that these factors can regulate cathelicidin expression (11). However, we hypothesized that by including 25D3 in culture at a concentration similar to that found in normal skin, TGF- $\beta_{1-}$ mediated induction of CYP27B1 could then lead to generation of $1,25 \mathrm{D} 3$ and the subsequent induction of cathelicidin, CD14, and TLR2. Neither 25D3 nor TGF- $\beta_{1}$ alone affected expression; however, their simultaneous addition induced cathelicidin, CD14, and TLR2 (Figure 4). Specific inhibition of CYP27B1 by itraconazole, or addition of the VDR antagonist ZK159222, blocked this effect (Figure 4). These data therefore show that the action of TGF- $\beta_{1}$ and $25 \mathrm{D} 3$ was due to the enzymatic generation of $1,25 \mathrm{D} 3$.

Induction of vitamin $D_{3}$-regulated immune function in skin wounds is mediated by CYP27B1 activation in vivo. To further investigate the significance of increased CYP27B1 activation in skin injury, wounds from mice lacking the CYP27B1 enzyme were evaluated for induction of an innate immune response. CD14 is regulated by $1,25 \mathrm{D} 3$ (29), and in mouse wounds, CD14 was induced in wild-type animals but not in those lacking CYP27B1 (Figure 5A). In contrast, murine Camp, which is not regulated by $1,25 \mathrm{D} 3$ (7), was increased in wounds in both wild-type and CYB27B1-deficient animals (Figure 5B). These data underscore the importance of CYP27B1activated $1,25 \mathrm{D} 3$ production to induce a vitamin $\mathrm{D}_{3}$-triggered immune response in skin injury.

1,25D3 enhances TLR2/6 function by keratinocytes. In order to explore the functional significance of TLR2 and CD14 induction by increased 1,25D3, keratinocytes were exposed to several TLR ligands, and the antimicrobial response was measured by evaluating the expression of cathelicidin. In contrast to cells grown under basal con- ditions, keratinocytes pretreated with low doses of 1,25D3 (0.1 nM for 24 hours) responded to Malp-2 but not to other TLR agonists such as LPS, CpG, 19-kDa lipopeptide, and flagellin (Figure 6A). In addition to Malp-2, other ligands of the TLR2/ 6 heterodimer such as the fungal cell wall component zymosan and the synthetic lipoprotein FSL-1 were also capable of inducing cathelicidin after keratinocytes were activated by 1,25D3 (100 nM; Figure 6B). At low concentrations of $1,25 \mathrm{D} 3(0.1 \mathrm{nM})$, the significance of the enhanced responsiveness became even more apparent, as evidenced by a dosedependent increase of cathelicidin expression of up to 60-fold greater than that seen in cells without 1,25D3 (Figure 6C).

In order to examine whether this increased responsiveness to TLR2/ 6 ligands reflects a direct action of 1,25D3 on TLR2 function or an indirect effect related to the changes in keratinocyte differ-
A

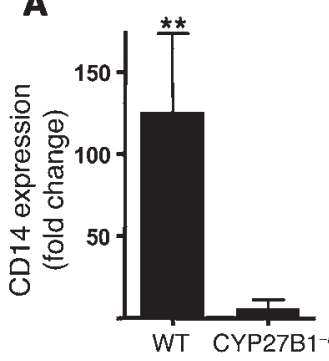

B

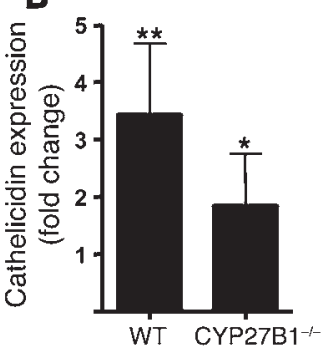

\section{Figure 5}

Activation of CYP27B1 is responsible for increased vitamin $D_{3}$ signaling in wounds. Wounds from CYP27B1-/- mice and age-matched wild-type littermates 24 hours after full-thickness sterile skin incision were evaluated for the expression of innate immune recognition and response molecules ( $n=5$ per group). Transcript abundance in wounded skin was measured by qPCR and normalized to noninjured skin from the same animal. (A) CD14 increased in wild-type animals after injury but not in mice lacking CYP27B1. (B) Cathelicidin was induced in wounds from both wild-type and CYP27B $1^{-1-}$ animals. Although cathelicidin induction was less in CYP27B1 $1^{-1}$ mice, the difference was not statistically significant. ${ }^{*} P<0.05,{ }^{* \star} P<0.01$ versus control uninjured skin, Mann-Whitney test. 
A

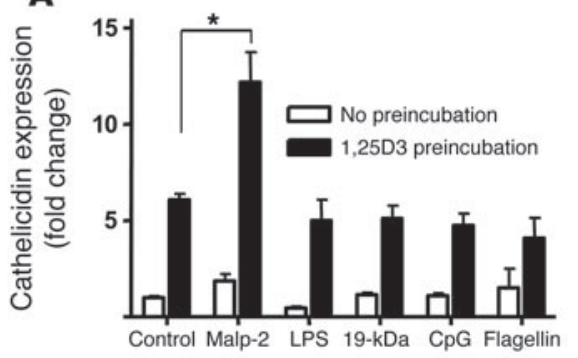

c

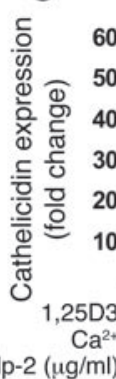

Malp-2

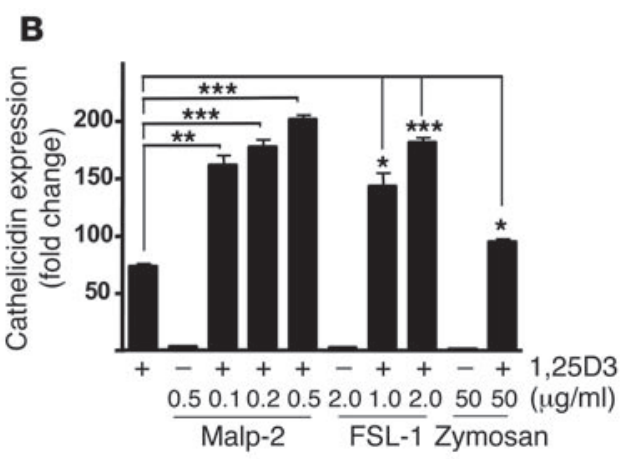

D

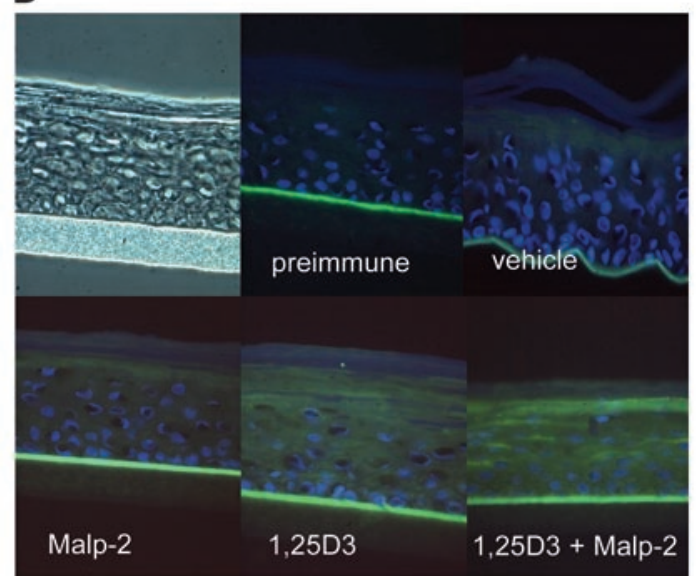

Figure 6

TLR2 function in keratinocytes is increased by 1,25D3. (A) To test the functional relevance of the increased expression of TLR2 and CD14 in keratinocytes exposed to 1,25D3, cells were incubated with a low dose of 1,25D3 (0.1 nM for 24 hours) and then stimulated with TLR ligands for an additional 24 hours. Cathelicidin mRNA abundance was measured as in Figure 2. Only TLR2/6 ligand Malp-2 increased expression above that induced by low-dose 1,25D3 alone. (B) Dose-dependent response of cathelicidin expression following administration of TLR2/6 agonists Malp-2, FSL-1, or zymosan at the indicated concentrations in the presence of 1,25D3 (100 nM). (C) Cathelicidin mRNA expression in keratinocytes incubated with a low dose of 1,25D3 $(0.1 \mathrm{nM})$ or a high calcium concentration $(1.7 \mathrm{mM})$ for 24 hours and then stimulated with Malp-2 for another 24 hours. (D) Organotypic epidermal constructs were stimulated with Malp-2 $(0.1 \mu \mathrm{g} / \mathrm{ml})$ alone or in the presence of 1,25D3 (100 $\mathrm{nM})$, and cathelicidin peptide expression was determined by immunostaining. Constructs were stained with a polyclonal anti-LL-37 antibody (green), and nuclei were detected with DAPI (blue). Original magnification, $\times 400$. (E) Cathelicidin mRNA expression was inhibited by a TLR2-neutralizing antibody applied to keratinocytes stimulated with Malp-2 or FSL-1 in the presence of 1,25D3 (100 nM). Data are mean \pm SD of a single experiment performed in triplicate and are representative of 3 independent experiments. ${ }^{*} P<0.05,{ }^{* *} P<0.01$, ${ }^{* *} P<0.001$, Student's $t$ test.

entiation induced by $1,25 \mathrm{D} 3$, keratinocytes were studied following differentiation induced by stratification at the air-liquid interface or by increasing the concentration of extracellular calcium. Cultured keratinocyte monolayers incubated with high calcium concentrations $(1.7 \mathrm{mM})$ as an alternative signal to induce terminal differentiation did not respond to TLR2/6 activation by Malp-2 (Figure 6C). This treatment effectively stimulated differentiation, as seen by increased expression of involucrin and altered cell morphology (data not shown). Similarly, differentiated keratinocytes in epidermal constructs did not respond to Malp-2 in the absence of 1,25D3 but did after its addition (Figure 6D). The response to Malp-2 was caused by TLR2, as a neutralizing antibody against TLR2 blocked the 1,25D3-dependent response to both Malp-2 and FSL-1 (Figure 6E). Also, in support of a direct effect of 1,25D3 on TLR2 function were observations of the transcriptional response of the human cathelicidin gene promoter to Malp-2. Keratinocytes were transfected with CAMP promoter constructs, including one with a site-directed disruption of the CAMP VDRE. In the presence of 1,25D3, promoter activity was responsive to Malp-2 only in constructs that included the VDRE (Figure 7A). Also, inhibition of the VDR with ZK159222 inhibited the capacity of Malp-2 to stimulate an increase in cathelicidin mRNA (Figure 7B). Thus, 1,25D3 both induces cathelicidin by itself and enables TLR2 to further increase expression of this AMP.

\section{Discussion}

Wounding creates a breach in the otherwise intact physical and chemical cutaneous barrier against the outer environment. Shortly after a wound is created, epithelial cells become activat- 
A

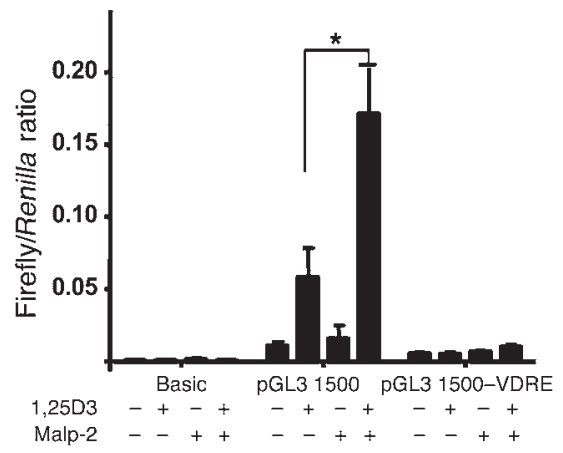

B

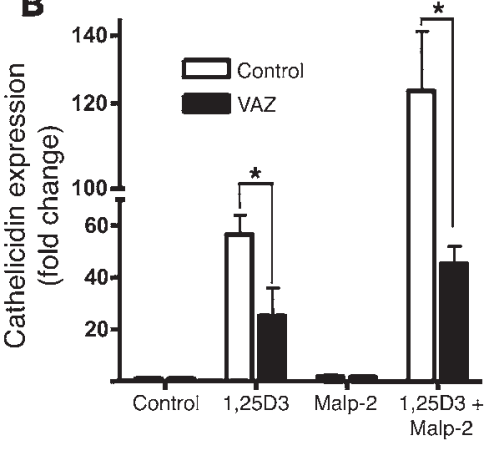

Figure 7

TLR2 activation signals cathelicidin induction through the vitamin $D_{3}$ pathway. (A) $A$ functional VDRE was required for transcriptional activation of cathelicidin by Malp-2 and 1,25D3. HaCaT keratinocytes containing cathelicidin promoter reporter constructs (pGL3 1,500) were treated with Malp-2 $(0.1 \mu \mathrm{g} / \mathrm{ml})$ in the presence or absence of 1,25D3 $(100 \mathrm{nM})$. Promoter constructs with a deleted VDRE at position $-619 \mathrm{bp}$ to $-633 \mathrm{bp}$ (pGL3 1,500-VDRE) lost transcriptional activity in all experiments. Values represent the ratio between firefly and Renilla luciferase activities. (B) In addition, treatment of keratinocytes with the VDR antagonist ZK159222 (10-7 M) blocked Malp-2-induced cathelicidin. Data are mean \pm SD of a single experiment performed in triplicate and are representative of 3 independent experiments. ${ }^{*} P<0.05$, Student's $t$ test.

ed, produce multiple factors to protect against microbial invasion, and trigger recruitment of leukocytes to further defend the wound. These epithelially derived molecules include AMPs, enzymes, cytokines, and growth factors that initiate a complex and incompletely understood process of repair. Only after several hours to days do keratinocytes from the edge of the wound begin to migrate and reepithelialize the injury, reestablishing an effective physical barrier. Prior to final formation of this barrier, it is essential for the unique wound micromilieu to protect the host from infection. Cathelicidin AMPs have been shown to be essential to this process (18), but to our knowledge the elements responsible for control of expression during injury were not previously known.

Recently, 1,25D3 was found to induce human CAMP expression in keratinocytes in vitro and in vivo (6-8), and an observed increase in cathelicidin expression after UVB irradiation was proposed to be caused by an increase in 1,25D3 (30). These observations led us to examine whether the regulation of vitamin $\mathrm{D}_{3}$ is involved in the wound repair response. We found multiple genes under the influence of vitamin $D_{3}$, and TLR2, which to our knowledge was not previously known to be inducible by $1,25 \mathrm{D} 3$, was induced in skin after injury. Our results suggest that soluble factors in the wound such as TGF- $\beta_{1}$ stimulate keratinocytes to increase the metabolic conversion of $25 \mathrm{D} 3$ to $1,25 \mathrm{D} 3$, thus driving the expression and function of human cathelicidin and a TLR2 complex. The increase in TLR2 enabled cells to respond to microbial stimulation and further enhance the AMP response if necessary, while also amplifying the generation of active vitamin $\mathrm{D}_{3}$. This elegant system of control of innate immunity by vitamin $\mathrm{D}_{3}$ was to our knowledge previously unknown and adds a new element to the understanding of innate immune defense during wound repair.

Keratinocytes possess the complete enzymatic machinery to produce active $1,25 \mathrm{D} 3$ from stored inactive proforms (31). The final step of endogenous activation of vitamin $\mathrm{D}_{3}$ is hydroxylation of 25D3 by CYP27B1. Cytokines such as IFN- $\gamma$ and TNF- $\alpha$ were found over a decade ago to induce 1,25D3 production in nondifferentiated keratinocytes $(27,28)$, but the relevance of this was unclear. Our results suggest that the induction of TLR2, CD14, and cathelicidin is a direct consequence of increased vitamin $\mathrm{D}_{3}$ metabolism. Furthermore, TGF- $\beta_{1}$ was found to increase CYP27B1 and subsequently induce local 1,25D3. Lacking 25D3 as a precursor substrate, inhibiting CYP27B1 enzymatic activity, and blocking the VDR all inhibited the capacity of TGF- $\beta_{1}$ to act in vitro. In vivo, mice lacking the CYP27B1 enzyme failed to respond to skin injury with an increase in 1,25D3-regulated CD14. Further investigations to confirm in vivo that inhibition of CYP27B1 also blocks the AMP response would be helpful, but are hindered by the lack of a VDRE in murine Camp (7). This distinct difference between humans and mice remains to be explained, but may reflect an evolutionary adaptation to the nocturnal nature of mice compared with humans. However, data derived from the results of topical administration of excess $1,25 \mathrm{D} 3$ to human volunteers did confirm that $1,25 \mathrm{D} 3$ can act in vivo to induce both TLR2 and cathelicidin (Figure 2D).

Our findings also show that activation of CYP27B1, and subsequent generation of $1,25 \mathrm{D} 3$, enables keratinocytes to respond to microbial components such as Malp-2 or zymosan. These observations complement, but are distinct from, recent work in monocytes showing that activation of TLR2 leads to an increase in $1,25 \mathrm{D} 3$ (4). One sees that an amplification loop is possible in skin that is not apparent in monocytes (Figure 8). 1,25D3 leads to increased TLR 2 in keratinocytes, and increased signaling by
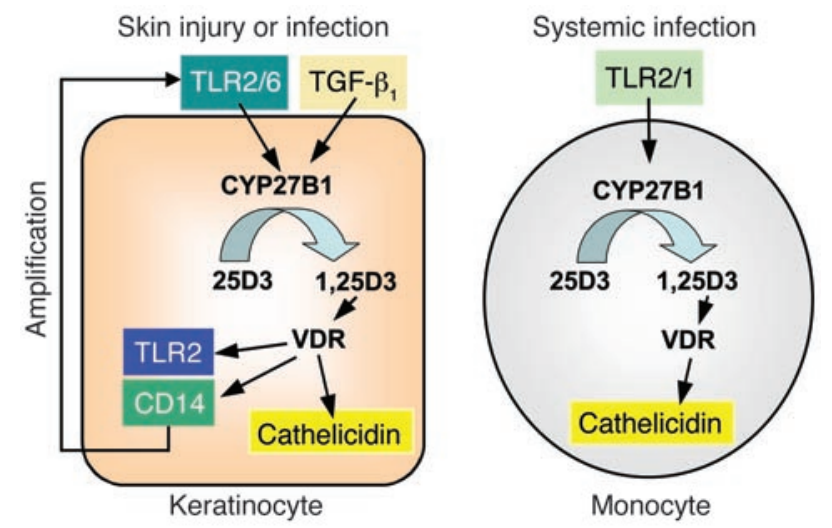

\section{Figure 8}

Schematic model for 1,25D3-regulated innate immune functions in keratinocytes and monocytes. Two distinct 1,25D3-dependent pathways in keratinocytes and monocytes are shown. In skin injury, keratinocytes are activated by TGF- $\beta_{1}$ or TLR2/ 6 ligands, which then leads to induction of CYP27B1. As a consequence 25D3 is converted to $1,25 \mathrm{D} 3$, which, upon activation of the VDR, induces cathelicidin, TLR2, and CD14. The 1,25D3-induced TLR2 enables the response of keratinocytes to TLR2 activation, resulting in further increased cathelicidin expression. In contrast, circulating monocytes are activated by TLR2/1 agonists. As a consequence, the genes encoding the VDR and CYP27B1 are induced. CYP27B1 converts 25D3 to 1,25D3 and subsequently increases cathelicidin. 
TLR2 leads to elevated 1,25D3. This does not appear to occur in monocytes and keratinocytes are specific to each cell type. In the epidermis, factors involved in the wound repair process such as TGF- $\beta_{1}$ trigger $1,25 \mathrm{D} 3$-dependent immune responses in keratinocytes. In monocytes, TLR2/1 activation increases 1,25D3dependent antimicrobial activity (4), but vitamin $\mathrm{D}_{3}$ inhibits TLR expression and triggers hyporesponsiveness to pathogen-associated molecular patterns (32). Thus, distinct effects on innate immune recognition and response are operative during infection of skin or when microbes come in contact with monocytes.

Future studies of TLR and AMP expression in keratinocytes must include constitutively present elements of the epidermal micromilieu such as precursor forms of vitamin $\mathrm{D}_{3}$ not typically present in keratinocyte culture systems. Without inclusion of 25D3, the function of CYP27B1 to generate 1,25D3 would not have been detected, and the capacity of TGF- $\beta_{1}$ or TLR 2 to influence cathelicidin expression would have been overlooked.

Our results show for the first time to our knowledge that vitamin $\mathrm{D}_{3}$ signaling is important to microbial recognition and the antimicrobial response during injury. Activated vitamin $\mathrm{D}_{3}$ generated in the healing process initiates an antimicrobial response and increased sensitivity to microbial challenge. The recognition receptors TLR 2 and CD14, which are induced by 1,25D3 are important for defense against a wide range of flora relevant to wound infections because they recognize elements of both Gram-negative and Gram-positive infections such as peptidoglycan and lipoteichoic acid (19), and heterodimers of TLR2 with TLR1 and TLR6 detect triacetylated and diacetylated lipopeptides, respectively $(33,34)$. In addition, TLR $2 / 6$ recognizes fungal wall and mycoplasma components. This is consistent with clinical observations that a large proportion of acute wounds show fungal colonization, yet do not show overt signs of fungal infection (35), and that cathelicidin is fungicidal (36). It will be important to investigate whether disturbed vitamin $\mathrm{D}_{3}$ metabolism following injury contributes to abnormal wound repair or infection, or whether other disorders characterized by altered innate immune responses may benefit from modification of vitamin $\mathrm{D}_{3}$ metabolism in skin.

\section{Methods}

Patients and treatments. In order to study human skin wounds, 5-mm fullthickness wounds were induced in volunteers $(n=5)$ under aseptic conditions with local anesthesia as described previously (18). At 24 hours after incision, 4-mm punch biopsies were taken from the site of injury and immediately fixed in formalin for immunostaining or frozen in liquid $\mathrm{N}_{2}$ for subsequent RNA isolation. Intact skin from healthy volunteers served as a control $(n=4)$. In order to examine the effects of topical $1,25 \mathrm{D} 3$, healthy skin of volunteers $(n=7)$ was treated once topically with $1,25 \mathrm{D} 3$ $(1.0 \mathrm{mM})$ and left under occlusion for 4 days. Vehicle-treated contralateral skin served as a control. After treatment, skin biopsies were obtained from both sites, fixed in $10 \%$ formalin, and embedded in paraffin for immunostaining. All treatments and sample acquisitions, including skin biopsies, were approved by the committees on investigations involving human subjects of the University of California, San Diego, and the University of Michigan. For all procedures, informed consent was obtained.

Animal studies. Skin wounds were collected from CYP27B1-deficient mice (37) or their wild-type littermates ( $n=5$ per group) 24 hours after induction of a $5-\mathrm{mm}$ full-thickness wound under aseptic conditions. Noninjured skin from the same animal served as control. Skin samples were immediately frozen for subsequent RNA isolation. All protocols used in the studies were approved by the committees on investigations involving animal subjects at the Veterans Affairs Medical Center, San Francisco, and the University of California, San Diego.

Cell culture and stimuli. Human keratinocytes were grown in serum-free EpiLife cell culture media (Cascade Biologics) containing $0.06 \mathrm{mM} \mathrm{Ca}^{2+}$ and $1 \times$ EpiLife Defined Growth Supplement (EDGS) at $37^{\circ} \mathrm{C}$ under standard tissue culture conditions. Stock cultures were maintained for up to 5 passages in this media with the addition of $50 \mathrm{U} / \mathrm{ml}$ penicillin and $50 \mu \mathrm{g} / \mathrm{ml}$ streptomycin. HaCaT keratinocytes were cultured in DMEM (Sigma-Aldrich) with 10\% FCS. Cells at 50\%-70\% confluence were stimulated in fresh medium after preincubation with 1,25D3 (0.1-100 nM for 4-24 hours; Sigma-Aldrich). Alternatively, normal human epidermal keratinocytes were stimulated in the presence of 1,25D3 (0.1-100 nM). Stimuli included TLR2/6 ligands Malp-2 (0.001-0.5 $\mu \mathrm{g} / \mathrm{ml}$; Alexis Biochemicals), FSL-1 (Pam2Cys-GDPKHPKSF, 1-2 $\mu \mathrm{g} / \mathrm{ml}$; Invivogen), and zymosan $(50 \mu \mathrm{g} / \mathrm{ml}$; Invivogen) as well as flagellin $(50 \mathrm{ng} / \mathrm{ml}$; Alexis Biochemicals), LPS ( $1 \mu \mathrm{g} / \mathrm{ml}$; Sigma-Aldrich), lipoteichoic acid (10 $\mu \mathrm{g} / \mathrm{ml}$; Sigma-Aldrich), 19-kDa lipopeptide (10 $\mu \mathrm{g} / \mathrm{ml}$, a kind gift from S. Krutzik, UCLA, Los Angeles, California, USA), CPG DNA (10 $\mu \mathrm{g} / \mathrm{ml}$; SigmaAldrich), $\mathrm{Ca}^{2+}(1.7 \mathrm{mM}), \mathrm{LPS}(1 \mu \mathrm{g} / \mathrm{ml}$; Sigma-Aldrich), TNF- $\alpha$ (20 ng/ml; Chemicon International), and TGF- $\beta_{1}(0.1-1 \mathrm{ng} / \mathrm{ml}$; R\&D Systems). A TLR2-blocking antibody (clone TL2.1, $5 \mu \mathrm{g} / \mathrm{ml}$; eBioscience) was used in order to investigate the role of TLR2 signaling. Furthermore, keratinocytes were pretreated with $10^{-7} \mathrm{M}$ of either the VDR antagonist ZK159222 or the CYP inhibitor itraconazole before stimulation with TGF- $\beta_{1}$ in the presence of 25D3 (10 nM; Sigma-Aldrich).

Organotypic culture. Epidermal human skin-like tissue constructs were obtained from MatTek Corp. and cultured according to manufacturer's instructions. Skin constructs were cultured in maintenance media (EPI-100-MM; MatTek Corp.) and stimulated with Malp-2 $(0.1 \mu \mathrm{g} / \mathrm{ml})$ for 24 hours in the presence of $1,25 \mathrm{D} 3(100 \mathrm{nM})$. Skin constructs were cut after stimulation and transferred to TRIzOL (Invitrogen) for RNA extraction or immediately frozen in Tissue-Tek (Sakura) for immunostaining.

Quantitative real-time RT-PCR. RNA was extracted from cells, epidermal constructs, or tissue using TRIzOL, and $1 \mu \mathrm{g}$ RNA was reverse transcribed using iScript (Bio-Rad). Expression of cathelicidin and GAPDH was analyzed by quantitative real-time RT-PCR (qPCR) as described previously (11). Predeveloped TaqMan assay probes (Applied Biosystems) were used for the analyses of the expression of CYP24A1, CYP27B1, CD14, CD36, TLR1, TLR2, TLR4, TLR6, and murine CD14. All analyses were performed in triplicate from 2-5 independent experiments in an ABI Prism 7000 Sequence Detection System (Applied Biosystems). Fold induction relative to the vehicle treated control in in vitro experiments was calculated using the comparative threshold cycle (Ct) method, where $\Delta \mathrm{Ct}$ is $\Delta \mathrm{Ct}_{\text {stimulant }}$ $\Delta \mathrm{Ct}_{\text {vehicle }}, \Delta \mathrm{Ct}$ is $\mathrm{Ct}_{\text {gene }}-\mathrm{Ct}_{\mathrm{GAPDH}}$, and $\mathrm{Ct}$ is the cycle at which an arbitrary detection threshold is crossed. For quantification of transcript abundance in tissue samples, target gene expression was normalized to GAPDH and compared with untreated or noninjured skin.

Promoter analysis and site-directed mutagenesis. To analyze transcriptional activity, a 1,500-bp fragment of the $5^{\prime}$ untranslated region of CAMP was amplified with primers 5 '-CACACAGCTAGCGGAACCCCTGGACAACGG-3' (pGL3 1,500; sense) and 5'-GAGAGACTCGAGGTCTGCCTCCCTCTAGCC-3' (pGL3 1,500; antisense) using human genomic DNA as a template. Primers were designed to introduce an NheI restriction site at the $5^{\prime}$ end and an XhoI restriction site at the $3^{\prime}$ end of the amplicon. The amplification product was cloned into the TOPO vector (Invitrogen) and transformed into One Shot TOP10 Electrocomp E. coli cells (Invitrogen). After DNA purification using the Wizard Plus, SV Miniprep purification system (Promega), the construct was digested with NheI and XhoI and then subcloned into the promoterless pGL3-basic 
firefly luciferase vector (Promega). The functional role of a previously described VDRE (6) for the transcription of CAMP was studied by sitedirected mutagenesis of the VDRE. The VDRE at position $-619 \mathrm{bp}$ to $-633 \mathrm{bp}$ relative to the translation start site was deleted with primers 5'-AACTTCTGCTTCAGTGATTCTCAT-3' (sense) and 5'-ATGAGAATCACTGAAGCAGAAGTT-3' (antisense) using a protocol published by Prinzen et al. (38). The resulting plasmid lacking the VDRE binding site cloned in the pGL3-basic vector was termed pGL3 1,500-VDRE. All resulting constructs were confirmed by sequencing. For transfection, $\mathrm{HaCaT}$ cells were seeded in 24-well plates (BD Biosciences) and used for transfection at 50\%-70\% confluence. Cells were transfected with CAMP reporter plasmids and $0.1 \mathrm{ng}$ of an internal control Renilla luciferase expression plasmid (Promega) by using $1.5 \mu$ l of transfection reagent FuGENE 6 (Roche Diagnostics) according to the manufacturer's instructions. Cells were stimulated with Malp-2 and 1,25D3 6 hours after transfection and incubated 24 hours before harvesting with $50 \mu \mathrm{l}$ of passive lysis buffer (Promega). Firefly luciferase activity from the CAMP pGL3 reporter vectors and Renilla luciferase activity were measured by the Dual Luciferase Assay system (Promega) in a luminometer (Optocomp I; MGM Instruments). Promoter activity was reported as the ratio between firefly and Renilla luciferase activities in each sample.

Immunostaining. After fixation in 2\% PFA and subsequent washings in PBS, sections from skin tissue or epidermal constructs were blocked in $3 \%$ BSA in PBS for 30 minutes at room temperature and stained with anti-cathelicidin LL-37 primary antibody or preimmune serum as described previously (39). After washings in PBS, slides from epidermal constructs were reprobed with an FITC-labeled goat anti-chicken antibody. Nuclei were detected with DAPI. After subsequent washings with PBS, slides were mounted in ProLong Anti-Fade reagent (Invitrogen) and evaluated with an Olympus BX41 microscope (Olympus). Paraffin-embedded, formalin-fixed tissue sections were rehydrated in a series of toluene, ethanol, and PBS. Endogenous peroxidase activity was quenched by 30 minutes incubation in $0.3 \% \mathrm{H}_{2} \mathrm{O}_{2}$ in water, and sections were microwaved for 4 minutes in antigen retrieval solution $(0.01 \mathrm{M}$ citric acid, $0.05 \mathrm{M} \mathrm{NaOH}, \mathrm{pH} 6.0$ ). Sections were blocked with $2 \%$ goat serum in PBS, $3 \%$ BSA, and then incubated with rabbit anti LL-37 (18) or anti-TLR2 (Abcam) primary antibody in PBS, $0.1 \%$ BSA. Sections were washed in PBS and detected with biotinylated secondary antibodies

(Vector Laboratories) and diaminobenzidine substrate (Sigma-Aldrich) per the manufacturers' instructions.

Western blot. Primary keratinocytes were stimulated with $1,25 \mathrm{D}_{3}$ $(100 \mathrm{nM})$ or vehicle for 24 hours and subsequently lysed in ice-cold lysis buffer (1\% Triton-X in PBS containing proteinase inhibitors). After centrifugation, equal amounts of protein were mixed with loading buffer (0.25 M Tris $\mathrm{HCl}, 10 \%$ SDS, $10 \%$ glycerol, and 5\% $\beta$-mercaptoethanol) and loaded onto a $10 \%$ Tris-Tricine gel (ISC Bioexpress). After separation, proteins were blotted onto a PVDF membrane (Millipore) and blocked in $5 \%$ milk (Bio-Rad) in TBS containing $0.1 \%$ Tween 20 for 1 hour at room temperature. After washings in TBS containing $0.1 \%$ Tween 20 , membranes were stained with a rabbit polyclonal anti-CD14 (Abcam) or an anti-TLR2 antibody (eBioscience), washed again in TBS containing $0.1 \%$ Tween 20, and reprobed with a HRP-coupled goat anti-rabbit antibody (Dako). Stained protein was visualized using the Western Lightning system (PerkinElmer) and quantified using ImageJ version $1.36 \mathrm{~b}$ software (http://rsb.info.nih.gov/ij/). Blots were reprobed with an anti- $\alpha$-tubulin antibody (Abcam) to ensure equal protein loading. Analyses from 3 independent experiments were performed.

Statistics. Differences between groups were compared using 2-tailed Student's $t$ test or Mann-Whitney test as indicated in the figure legends. A $P$ value less than 0.05 was considered significant.

\section{Acknowledgments}

This work was supported by a VA Merit award and NIH grants NIH/NIAID HHSN26620040029C, ADB contract no. N01-AI40029AI48176, AI052453, and AR45676 to R.L. Gallo, by grant BU 2212/1-1 from the Deutsche Forschungsgemeinschaft to A.S. Büchau, and by grant BMBF-LPD 9901/8-119 from the Deutsche Akademie der Naturforscher Leopoldina to J. Schauber.

Received for publication August 22, 2006, and accepted in revised form December 12, 2006.

Address correspondence to: Richard L. Gallo, 3350 La Jolla Village Drive, Mail Code 151, San Diego, California 92161, USA. Phone: (858) 552-8585 ext. 6149; Fax: (858) 552-7436; E-mail: rgallo@ucsd.edu.

1. Roth, D.E., et al. 2004. Association between vitamin $\mathrm{D}$ receptor gene polymorphisms and response to treatment of pulmonary tuberculosis. J. Infect. Dis. 190:920-927.

2. Wayse, V., Yousafzai, A., Mogale, K., and Filteau, S. 2004. Association of subclinical vitamin D deficiency with severe acute lower respiratory infection in Indian children under 5 y. Eur. J. Clin. Nutr. 58:563-567.

3. Barnes, P.F., Modlin, R.L., Bikle, D.D., and Adams, J.S. 1989. Transpleural gradient of 1,25-dihydroxyvitamin D in tuberculous pleuritis. J. Clin. Invest. 83:1527-1532.

4. Liu, P.T., et al. 2006. Toll-like receptor triggering of a vitamin D-mediated human antimicrobial response. Science. 311:1770-1773.

5. Zasloff, M. 2006. Fighting infections with vitamin D. Nat. Med. 12:388-390.

6. Wang, T.-T., et al. 2004. Cutting edge: 1,25-dihydroxyvitamin D3 is a direct inducer of antimicrobial peptide gene expression. J. Immunol. 173:2909-2912.

7. Gombart, A.F., Borregaard, N., and Koeffler, H.P. 2005. Human cathelicidin antimicrobial peptide (CAMP) gene is a direct target of the vitamin D receptor and is strongly up-regulated in myeloid cells by 1,25-dihydroxyvitamin D3. FASEB J. 19:1067-1077.
8. Weber, G., et al. 2005. Vitamin D induces the antimicrobial protein hCAP18 in human skin. J. Invest. Dermatol. 124:1080-1082.

9. Bikle, D.D., Oda, Y., and Xie, Z. 2004. Calcium and 1,25(OH)2D: interacting drivers of epidermal differentiation. J. Steroid Biochem. Mol. Biol. 89-90:355-360.

10. Tian, X.Q., Chen, T.C., and Holick, M.F. 1995. 1,25dihydroxyvitamin D3: a novel agent for enhancing wound healing. J. Cell. Biochem. 59:53-56.

11. Schauber, J., Dorschner, R., Yamasaki, K., Brouha, B., and Gallo, R. 2006. Control of the innate epithelial antimicrobial response is cell-type specific and dependent on relevant microenvironmental stimuli. Immunology. 118:509-519.

12. Zaiou, M., and Gallo, R.L. 2002. Cathelicidins, essential gene-encoded mammalian antibiotics. J. Mol. Med. 80:549-561.

13. Nizet, V., et al. 2001. Innate antimicrobial peptide protects the skin from invasive bacterial infection. Nature. 414:454-457.

14. Chromek, M., et al. 2006. The antimicrobial peptide cathelicidin protects the urinary tract against invasive bacterial infection. Nat. Med. 12:636-641.

15. Pütsep, K., Carlsson, G., Boman, H., and Andersson, M. 2002. Deficiency of antibacterial peptides in patients with morbus Kostmann: an observation study. Lancet. 360:1144-1149.

16. Brown, A.J., Dusso, A., and Slatopolsky, E. 1999.
Vitamin D. Am. J. Physiol. 277:F157-F175

17. Overbergh, L., et al. 2004. Regulation of 25hydroxyvitamin d-1alpha-hydroxylase by IFNgamma in human monocytic THP1 cells. J. Steroid Biochem. Mol. Biol. 89-90:453-455.

18. Dorschner, R.A., et al. 2001. Cutaneous injury induces the release of cathelicidin anti-microbial peptides active against group A Streptococcus. J. Invest. Dermatol. 117:91-97.

19. Akira, S., Uematsu, S., and Takeuchi, O. 2006. Pathogen recognition and innate immunity. Cell. 124:783-801.

20. Braff, M.H., Bardan, A., Nizet, V., and Gallo, R.L. 2005. Cutaneous defense mechanisms by antimicrobial peptides. J. Invest. Dermatol. 125:9-13.

21. Zhang, D.E., Hetherington, C.J., Gonzalez, D.A., Chen, H.M., and Tenen, D.G. 1994. Regulation of CD14 expression during monocytic differentiation induced with 1 alpha,25-dihydroxyvitamin D3. J. Immunol. 153:3276-3284.

22. Prosser, D.E., and Jones, G. 2004. Enzymes involved in the activation and inactivation of vitamin $\mathrm{D}$. Trends Biochem. Sci. 29:664-673.

23. Lehmann, B., Genehr, T., Knuschke, P., Pietzsch, J., and Meurer, M. 2001. UVB-induced conversion of 7-dehydrocholesterol to 1alpha,25-dihydroxyvitamin D3 in an in vitro human skin equivalent. J. Invest. Dermatol. 117:1179-1185. 
24. Koeffler, H.P., Reichel, H., Bishop, J.E., and Norman, A.W. 1985. gamma-Interferon stimulates production of 1,25-dihydroxyvitamin D3 by normal human macrophages. Biochem. Biophys. Res. Commun. 127:596-603.

25. Stoffels, K., et al. 2006. Immune regulation of 25 hydroxyvitamin-D3-1alpha-hydroxylase in human monocytes. J. Bone Miner. Res. 21:37-47.

26. Dusso, A.S., et al. 1997. gamma-Interferon-induced resistance to 1,25-(OH)2 D3 in human monocytes and macrophages: a mechanism for the hypercalcemia of various granulomatoses. J. Clin. Endocrinol. Metab. 82:2222-2232.

27. Bikle, D.D., Pillai, S., Gee, E., and Hincenbergs, M. 1989. Regulation of 1,25-dihydroxyvitamin D production in human keratinocytes by interferongamma. Endocrinology. 124:655-660.

28. Bikle, D.D., Pillai, S., Gee, E., and Hincenbergs, M. 1991. Tumor necrosis factor-alpha regulation of 1,25-dihydroxyvitamin D production by human keratinocytes. Endocrinology. 129:33-38.

29. Matsuura, K., et al. 1992. Identification of a tissue-specific regulatory element within the murine CD14 gene. J. Biol. Chem. 267:21787-21794.

30. Mallbris, L., Edstrom, D.W., Sundblad, L., Granath, F., and Stahle, M. 2005. UVB upregulates the antimicrobial protein hCAP18 mRNA in human skin. J. Invest. Dermatol. 125:1072-1074.

31. Vantieghem, K., Kissmeyer, A.M., De Haes, P., Bouillon, R., and Segaert, S. 2006. UVB-induced production of 1,25-dihydroxyvitamin D3 and vitamin D activity in human keratinocytes pretreated with a sterol Delta7-reductase inhibitor. J. Cell. Biochem. 98:81-92.

32. Sadeghi, K., et al. 2006. Vitamin D3 down-regulates monocyte TLR expression and triggers hyporesponsiveness to pathogen-associated molecular patterns. Eur. J. Immunol. 36:361-370.

33. Jiang, Z., et al. 2005. CD14 is required for MyD88independent LPS signaling. Nat. Immunol. 6:565-570.
34. Hoebe, K., et al. 2005. CD36 is a sensor of diacylglycerides. Nature. 433:523-527.

35. Vindenes, H., and Bjerknes, R. 1995. Microbial colonization of large wounds. Burns. 21:575-579.

36. Lopez-Garcia, B., Lee, P.H., Yamasaki, K., and Gallo, R.L. 2005. Anti-fungal activity of cathelicidins and their potential role in Candida albicans skin infection. J. Invest. Dermatol. 125:108-115.

37. Bikle, D., et al. 2004. Mice lacking 25OHD 1alphahydroxylase demonstrate decreased epidermal differentiation and barrier function. J. Steroid Biochem. Mol. Biol. 89-90:347-353.

38. Prinzen, C., Muller, U., Endres, K., Fahrenholz, F., and Postina, R. 2005. Genomic structure and functional characterization of the human ADAM10 promoter. FASEB J. 19:1522-1524.

39. Braff, M.H., Zaiou, M., Fierer, J., Nizet, V., and Gallo, R.L. 2005. Keratinocyte production of cathelicidin provides direct activity against bacterial skin pathogens. Infect. Immun. 73:6771-6781. 